\title{
NO HAY DOLOR PEQUEÑO. \\ LA RESPUESTA DEL ESTADO \\ COLOMBIANO FRENTE A LOS \\ DAÑOS SUFRIDOS POR LA NIÑEZ Y LA ADOLESCENCIA VÍCTIMAS DEL CONFLICTO ARMADO ${ }^{1}$
}

\author{
Margarita Jaimes Velásquez ${ }^{2}$ \\ Cindy Hernández ${ }^{3}$ \\ Katia Palencia Sánchez ${ }^{4}$
}

\section{Resumen}

La nación colombiana ha estado sumergida en un conflicto armado caracterizado por sus diversas expresiones de violencias, por su extensión en el tiempo y la geografía. Así las cosas, las afectaciones sufridas por las víctimas, no siempre fueron experimentadas de igual manera por parecidos que fueran los hechos, de manera que los impactos en la vida y la siquis de las personas también fueron

\footnotetext{
$1 \quad$ El presente artículo científico es resultado de la investigación "La Reparación Integral a Los Niños, Niñas Y Adolescentes Víctimas del Conflicto Armado ¿Una Utopía”, financiada por Colciencias y la Corporación Universitaria del Caribe CECAR, dirigida por la investigadora Margarita Jaimes

2 Abogada de la Universidad del Atlántico, doctoranda en Política y Gobierno de la Universidad Católica de Córdoba (Argentina.) Docente investigadora adscrita a GICSER en la línea de Derechos Humanos y DIH, de la Facultad de Derecho de la Corporación Universitaria del Caribe-CECAR.Cel 3006776424 margarita.jaimes@cecar.edu.co 3 Joven investigadora de Colciencias de la convocatoria $N^{\circ} 617$, Ejecutora del proyecto de investigación La Reparación Integral A Los Niños, Niñas Y Adolescentes Víctimas Del Conflicto Armado ¿Una Utopía?, en la modalidad de Beca - Pasantía; adscrita al grupo de GISER de la corporación universitaria del caribe CECAR.

4 Abogada de la Universidad del Norte, doctoranda en Ciencias Políticas, Administración Pública y Relaciones Internacionales de la Universidad Complutense de Madrid. Directora de Consultorio Jurídico y Centro de Conciliación de la Corporación Universitaria del Caribe- CECAR. Docente investigadora adscrita a GICSER en la línea de Derecho Público de la Facultad de Derecho de la Corporación Universitaria del Caribe-CECAR. Cel 3135358219. Katia.palencias@cecar.edu.co.
} 
diferenciales.Si bien, los proyectos de vida se transforman a lo largo de la vida, la victimización sufrida puede cambiar radicalmente el horizonte trazado de cualquier persona, máxime cuando se encuentra en la niñez o la adolescencia. En efecto, los daños en la construcción de la identidad producto de los trastornos de ánimo y de la incomprensión de los hechos en la vida de los niños, niñas y adolescentes (en adelante NNA) dificultan seriamente el ciclo vital en que se encuentran. Los Principios internacionales sobre la lucha contra la impunidad y los Principios Internacionales sobre el derecho de las víctimas a obtener reparaciones reafirman las obligaciones de los estados en la garantía de los Derechos Humanos. Así mismo, ratifican que la reparación tiene por objeto minimizar el impacto lesivo en la victima y restituirla, en la medida de lo posible, al estado anterior.

Palabras clave: Niños, niñas y adolescentes, victimas, enfoque diferencial, atención psicosocial

\section{Abstract}

The Colombian nation has been submerged in an armed conflict characterized by its diverse expressions of violence, by its extension in time and geography. Although life projects are transformed throughout life, the victimization suffered can radically change the horizon of any person, especially when they are in childhood or adolescence. In fact, the damage to the construction of identity caused by mood disorders and the incomprehension of the facts in the lives of children and adolescents (hereinafter NNA) seriously hampers the life cycle in which they find themselves. The International Principles on the Fight against Impunity and the International Principles on the Right of Victims to Reparations reaffirm the obligations of states to guarantee human rights. They also ratify that reparation is intended to minimize the harmful impact on the victim and to restore it, as far as possible, to the previous state.

Keywords: Children and adolescents, victims, differential approach, psychosocial care 


\section{Introducción}

Atendiendo lo anterior, se propone este escrito analizar las obligaciones del Estado Colombiano en materia de reparaciones psicosocial y examinar a la luz de la normatividad existente el cumplimiento de dichas obligaciones a través del programa de atenciones psicosocial para víctimas en el restablecimiento de los derechos de los niños, niñas y adolescentes. Para ello se tomarán los resultados obtenidos en la investigación realizada en Sincelejo titulada "La reparación integral a los niños, niñas y adolescentes víctimas del conflicto armado ¿una utopía?" realizada con niños y niñas entre los 12 y 18 años en la ciudad de Sincelejo.

\section{El impacto diferencial del conflicto en niños, niñas y adolescentes}

El conflicto armado visto desde sus efectos en la vida de las víctimas se explica en las siguientes palabras.

(...) uno de los escenarios que marca de manera más profunda a los individuos y a las sociedades. La irracionalidad y deshumanización de la guerra expone a las personas y a las comunidades a situaciones límite de violencia que generan en ellos procesos de deconstrucción y ruptura del tejido social, de pérdida del bienestar emocional y destrucción de sus proyectos de vida (Nación, 2009, p. 22).

Ahora, el conflicto armado interno afecta diferencialmente a la población, las tipologías de afectaciones están relacionadas al grupo etario, el sexo, la identidad o la orientación sexual, la etnia y la condición económica por mencionar solo algunos. Para el caso de la niñez y la adolescencia víctimas del conflicto la Defensoría del Pueblo en el 2003 (citado en Toro, 2011), expone que:

La situación de los niños en el conflicto armado colombiano no es distinta de la situación de los niños involucrados en otros conflictos armados. Para infortunio de Colombia, los grupos insurgentes y de autodefensa han abusado y continúan abusando de la vulnerabilidad y fragilidad de los niños. Día a día, los actores armados reclutan menores de 18 años, los utilizan como carne de cañón o carnada para infligir daños a sus enemigos. Cientos 
son desplazados. Las niñas no solamente son las compañeras sexuales de los irregulares, sino que son forzadas a prostituirse.

Además de las tipologías específicas sufridas en razón al ciclo vital en que se encuentran, se debe tener en cuenta que, frente a un mismo hecho violento, el impacto en la vida de los NNA será diferente y potencialmente más dañino que al resto de víctimas adultas, dada su condición de sujeto en proceso de formación del carácter, la identidad, la cosmovisión del universo que le rodea y la escasa comprensión de los problemas políticos y sociales que vive el país.

Su desarrollo armónico se afecta gravemente en tanto, el proyecto de vida individual y familiar se trunca abruptamente exponiéndolos a situaciones desconocidas y, en la mayoría de los casos, hostiles. Lo que puede provocar cambios conductuales y emocionales en la vida de los infantes y adolescentes con repercusiones a lo largo de la vida del individuo. Una inadecuada intervención psicológica y psicosocial o la inexistencia de ella, puede influir negativamente en el desarrollo de las habilidades sociales y familiares, haciéndoles difícil o traumáticas las relaciones interpersonales.
Al respecto, Hewitt. N, Gantiva. C, Vera. A, Cuervo. M \& Hernández. N. (2014) expresaron que los desórdenes en la salud mental producto de la exposición a situaciones traumáticas en el marco de la guerra suelen perdurar y afectar el desarrollo de los NNA. Explican que este grupo etario es particularmente vulnerable en situaciones de conflicto armado (Haroz et al., 2013) debido a su condición de individuo en proceso de crecimiento y desarrollo debido a que su personalidad y sistema de valores se encuentran en construcción (Pereda, 2012).

Afirman Hewitt, et al, (2014) que existen tipologías de afectaciones producto de la violencia como son: dificultades en el desarrollo (Mels, 2012; Murthy \& Lakshminarayana, 2006), sentimientos de culpa, inseguridad, agresividad, aislamiento, desesperanza, fragilidad emocional, trastornos del sueño, ansiedad y depresión que además pueden crear ideas distorsionadas sobre lo ocurrido (Cohen \& Mannarino, 2008; López-Navarrete et al., 2007; Pereda 2012). Vale aclarar que estas tipologías dependen de la edad y las capacidades de afrontamiento del resto de la familia. 
En el mismo sentido, los hábitos y costumbres también son involuntariamente modificados; en relación a esto, Ibáñez \& Díaz 2010, (citado en Toro, 2011) sostienen que la desestructuración de la cotidianidad y la inseguridad sobre el futuro generada por el cambio brusco que trastoca el proyecto de vida familiar e individual pone en cuestión el sistema de valores ante la necesidad de sobrevivir.

De otro lado, los impactos psicosociales identificados por los autores se concentran en daños existenciales que inciden en el proceso identitario del individuo, reflejándose en crisis respecto al ejercicio de la autonomía, daños psicosomáticos y daños colectivos o socioculturales (Bello M. N., 2009)

En ese mismo sentido La Corporación AVRE (2006) plantea, que los impactos psicosociales de la violencia sociopolítica se clasifican a nivel individual, familiar y comunicativo, los cuales pueden expresarse:

(...) en las diversas áreas psíquicas de las personas, pero el grado de compromiso de esas áreas depende de la naturaleza del daño y de las condiciones particulares de cada individuo. Se pueden detectar alteraciones en la actitud, actividad y comportamiento, en las funciones cognoscitivas (memoria, pensamiento, capacidad para concentrarse, capacidad para tomar decisiones), alteraciones en el estado de ánimo, el sueño, la alimentación, la sexualidad; ansiedad, y en algunos casos, desorganización severa de la conducta y de las funciones mentales.

Y agrega que:

Un mismo hecho de violencia es significado de manera diferente por niños, niñas, jóvenes, hombres y mujeres, adultos y personas mayores. Losimpactos del daño generan necesidades particulares de acuerdo al grupo generacional. ¿Qué ha pasado con esos niños que presenciaron la muerte de sus padres? También los han matado. Algunos han intentado suicidarse (...) todos ellos son personas que no han tenido asistencia psicológica. Es gente que no ha hecho el duelo. (...) siempre pensando en sus seres queridos, sin encontrar ocasión para llorar, deseando contar públicamentelaverdad delos hechos paraborrarlo que Diferentes experiencias muestran que en ciertas ocasiones, y cuando sucede un hecho de violencia extremo, la reacción de ciertas personas 
muestra comportamientos y actitudes como "sin razón", o "no normales"; lo cual puede evidenciar un trastorno mental: el daño en estas circunstancias puede ser severo y afectar toda la integridad y capacidad de la persona.

Al revisar los datos en la Red Nacional de Información- RNI- en Colombia hay 8. 356.734 de víctimas registradas de las cuales 2.313.360 son niños y niñas entre los Cero (0) y los dieciocho (18) años. Estas cifras demuestranqueel problemanoesmenor, porloqueelEstadocolombianodebe propender por garantizar el restablecimiento de sus derechos y garantizar las medidas de reparación integral y las garantías de no repetición.

En aras de resolver la problemática identificada con la niñez y la adolescencia en situación de desplazamiento, la Corte Constitucional en el Auto 251 de 2008 expresó que:

Los problemas deíndole psicosocial, hacen parte delos problemas transversales diferenciados que afecta a la niñez y adolescencia en situación de desplazamiento, planteando que a circunstancias del desplazamiento forzado genera un impacto destructivo sobre su estado de salud psicológica y emocional e influyendo negativamente en las etapas de maduración individual; ocasionando también dificultades cognitivas y emocionales (tales como desconcentración, desmotivación y tristeza) y en barreras a la socialización.

Cada uno de estos impactos afecta de distinta manera al individuo en crecimiento por lo que la atención integral debe siempre responder a la afectación particular de cada persona y a sus necesidades. Por tanto, la afectacion psicosocial y psicológica no solo debe ser diferenciada en razón a las necesidades explicitas del grupo poblacional, sino que se hace necesario la implementacion de un enfoque particular que ahonde en las afectaciones individuales del ser.

\section{El derecho a la reparación}

La reparación es un principio fundamental del derecho de gentes (CoIDH, 1993) que fue definido por la extinta Comisión Nacional de Reparación CNRR (2007) como "un proceso que busca dignificar a las victimas, mediante medidas que alivien su sufrimiento, compensen sus 
perdidas sociales, morales y materiales que han sufrido y restituyan sus derechos ciudadanos". La reparación es entonces, un proceso que supone la inversión de recursos humanos, teóricos, técnicos y económicos a fin de alcanzar el restablecimiento de los derechos conculcados a las victimas, poniendo especial interés en las tipologías de daño que puede sufrir una persona por un hecho violento.

Los Principios y directrices básicos sobre el derecho de las víctimas de violaciones manifiestas de las normas internacionales de derechos humanos y de violaciones graves del derecho internacional humanitario a interponer recursos y obtener reparaciones (ONU, 2005) establece que la reparación debe ser proporcional al daño causado. De igual manera, establece en cabeza del Estado la obligación de reparar sin perjucio de las acciones de repetición a que haya lugar contra el responsable de la violación. Además recuerda que, las medidas de reparación deben ajustarse a los derechos humanos a la igualdad y no discriminación establece además que dichos programas y procedimientos deben ser divulgados y publicitados ampliamente a fin de garantizar el acceso efectivo de toda la población afectada.

Los programas de reparaciones deben ser incluyentes y lo más completo posible a fin de incluir la mayor cantidad de violaciones a los derechos humanos posibles, poniendo especial atención en las victimas con antecedentes de exclusión, discriminación formal y material o marginadas en razón a su edad, su identidad y orientación sexual, su pertenencia a una etnia, su sexo o su condición social. Así lo advirtió la señora Diane Orentlicher, experta independiente encargada de actualizar el Conjunto de principios para la lucha contra la impunidad en su informe presentado en el año 2005 al Consejo Económico y Social de las Naciones Unidas (ECOSOC), cuando dice que las reparaciones no deben limitar las categorias de delitos a ser reparados en un programa de reparaciones, puesto que al hacer esto se impide el acceso a la justicia de un gran número de victimas, que se sentiran excluidas y por tanto un problema político sin resolver

Lo anterior implica para el caso Colombiano, identificar las diversas expresiones de violencia, los derechos que con ellas se vulneraron y los efectos en la vida de las victimas. Para garantizar un adecuado acceso y el restablecimiento de los derechos conculcados es necesario tener en cuenta el ciclo vital, el sexo, la etnia y la condición económica o laboral al momento de los hechos y al momento de aplicar el programa o las acciones de 
reparación. Ahora, solo se han mencionado algunas varibles de las muchas que se entrecruzan en la complejidad humana y en las propias dinamicas del conflicto armado interno.

Según la legislación interna, específicamente, la (Ley 1448 de 2011) tiene por objeto:

(...)establecerunconjuntodemedidasjudiciales, administrativas, sociales y económicas, individuales y colectivas, en beneficio de las víctimas de las violaciones contempladas en el artículo $3^{\circ}$ de la presente ley, dentro de un marco de justicia transicional, que posibiliten hacer efectivo el goce de sus derechos a la verdad, la justicia y la reparación con garantía de no repetición, de modo que se reconozca su condición de víctimas y se dignifique a través de la materialización de sus derechos constitucionales (Articulo 1)

Lo anterior quiere decir que, la reparación integral debe ser adecuada, dicho en otras palabras, que satisfaga las realidades y necesidades de las victimas; debeser transformadora, lo que implicaademas del restablecimiento de los derechos, cambios de actitud y aptitud cuando el caso así lo requiera, pero además debe ser efectiva y diferenciada, es decir, proporcional al daño causado y atendiendo a los impactos y particularidades de cada victima; entendiendo que algunas tienen protección constitucional reforzada.

Ahora, tal como lo establecen los instrumentos internacionales sobre la materia, la reparación integral debe comprender además de las medidas de indemnización, la restitución, la rehabilitación, la satisfacción y las garantías de no repetición en sus dimensiones individual, colectiva, material, moral y simbólica (articulo 5).

\section{La rehabilitación como medida de reparación}

Según los Criterios de Reparación y de Proporcionalidad Restaurativa de la CNRR (2007) "La rehabilitación busca restablecer la integridad física, psicológica, moral, legal ocupacional de las víctimas" a fin de restablecer sus derechos y su dignidad teniendo en cuenta los hechos victimizantes, las circunstancias y necesidades específicas de cada víctima.

Para la Organización Mundial de la Salud, en el Segundo Informe de su Comité Experto en Rehabilitación Médica (OMS, 1968). 
Es la aplicación coordinada de un conjunto de medidas médicas, sociales, educativas y profesionales para reparar y readaptar al individuo con el objeto de que alcance la mayor proporción posible de capacidad funcional". Distinguiendo tres tipos de rehabilitación, la médica, la social y la vocacional.

Si bien estas definiciones no explican cómo se materializan las medidas de rehabilitación, en la práctica se entiende como la provisión de servicios médicos, terapéuticosy otro tipo de servicios que permitan el restablecimiento de la integridad personal y el goce efectivo de derechos de las víctimas. Por ejemplo, (Corte Constitucional Sentencia T 045/10 de, 2009), plantea que dentro de los servicios de salud se debe incluir la atención psicológica y psiquiátrica especializada, argumentando que:

La necesidad de prestar atención psicológica y psiquiátrica a las víctimas del conflicto armado se desprende de las serias afectaciones en la salud y daños graves a la integridad mental que asumen distintas particularidades dependiendo del contexto social y cultural de la persona. La salud mental abarca -entre otros aspectos- el bienestar subjetivo, la percepción de la propia eficacia, la autonomía, la competencia, la dependencia intergeneracional y la autorrealización de las capacidades intelectuales y emocionales. En virtud de lo anterior, se requiere de una atención en salud mental que permita su recuperación, dentro de la que debe contemplarse la necesidad de romper el aislamiento, reconocer sus experiencias de miedo, identificar sus enfermedades, permitirles hablar de sus sentimientos de vergüenza y de culpa y romper los tabúes que los alimentan. Y, lo más importante, permitirles hablar de lo que vivieron y facilitar la reflexión en la comunidad, combatiendo la estigmatización y promoviendoactitudes positivas que permitan el reconocimiento de las víctimas.

Todo lo anterior, es recogido en el capítulo VIII de la (Ley 1448 de 2011), relativo a las medidas de rehabilitación, cuando indica que estas son "El conjunto de estrategias, planes, programas y acciones de carácter jurídico, médico, psicológico y social, dirigidos al restablecimiento de las condiciones físicas y psicosociales de las víctimas en los términos de la presente Ley" (Artículo 135). 
Para cumplir esta aspiracion el Estado ha diseñado y, se encuentra ejecutando, un programa de rehabilitación que incluye medidas individuales y colectivas liderado por el Ministerio de la Salud y la Protección Social a través del Plan Nacional para la Atención y Reparación Integral a las Víctimas. (Artículos 136 y 137) en el que confluyen muchas instituciones del orden nacional, regional y local, con ordenes precisas y especificas.

\section{El enfoque diferencial en la reparación integral}

El estado social de derecho tiene varios principios y valores en los que el eje central es la persona humana, algunos de ellos, como la participación y la dignidad humana adquieren mayor relevancia en situaciones que ponen en riesgo los derechos de personas de especial protección constitucional. Si bien todas las personas son iguales ante la Ley y el Estado, no es menos cierto que algunos grupos poblacionales han sufrido la exclusión histórica y sus derechos invisibilidades frente al resto de la nación. Así las cosas, los niños, las niñas, los adolescentes, las mujeres, las personas con orientaciones sexuales no hegemónicas, los grupos étnicos y los campesinos tienen riesgos específicos en razón a sus particularidades, por ello, la atención otorgada debe atender esas diferencias.

Según el Observatorio de Construcción de paz de la Universidad Jorge Tadeo Lozano, el enfoque diferencial "nace con la pretensión de ofrecer un panorama de restitución de derechos a víctimas del conflicto desde sus particularidades" ; así mismo, plantea que este enfoque "busca orientar la acción del Estado al otorgamiento de bienes y servicios a partir de las diferencias de los grupos poblacionales que habitan en su territorio" diferencias que se pueden identificar según en el género, edad, diversidad cultural y étnica, situación de discapacidad

Partiendo de esta premisa, "El enfoque diferencial permite visibilizar las vulnerabilidades y vulneraciones específicas de grupos e individuos; Partiendo del reconocimiento de la diferencia busca garantizar el principio de equidad" (ANSPE, 2013);

Este enfoque orienta las acciones del Estado a partir del otorgamiento de bienes y servicios de acuerdo a las necesidades y especificidades de cada grupo poblacional, es decir, 
Busca visibilizar vulnerabilidades y vulneraciones específicas de grupos e individuos específicos, y prioriza acciones de protección y restauración de los derechos vulnerados. Implica: identificar los vacíos y riesgos de protección de cada grupo y desarrollar herramientas para dar soluciones, promover la participación equitativa y planear y ejecutar medidas afirmativas basadas en caracterizaciones sistemáticas para la garantía del goce efectivo de los derechos de los diferentes grupos poblacionales" Acnur, 2008, (citado en Ministerio de la Protección Social, 2011: 27).

A su vez, la (Ley 1448 de 2011), lo reconoce como un principio de orientación y aplicación en las fases de atención, asistencia y reparación cuando expresa que se aplicara a todas las personas "con características particulares en razón de su edad, género, orientación sexual y situación de discapacidad" (Artículo 13) fin de actuar directamente sobre esas particularidades y necesidades específicas.

En este contexto, la atención a las victimas del conflicto armado es un problema social y público que demanda una intevención del Estado eficaz y efectiva, haciendo uso de toda su capacidad y de sus recursos para garantizar el restablecimiento desde perspectivas diferenciales y con de derechos y psicosocial. Así las cosas, la salud mental y psicosocial de los NNA victimas es una prioridad que no admite demoras, ni errores en el diseño de las acciones propuestas para mitigar su sufrimiento

\section{Enfoque Psicosocial}

Las consecuencias psicosociales producto de las violencias en el marco del conflicto armado, a pesar de ser sufridas por la víctima se reflejan en la sociedad, en tanto sujetos que integran un colectivo. Por ello, el estudio de estas afectaciones debe ser especializado para que se puedan analizar en su complejidad y en las variables diferenciales a fin de diseñar e implementar programas de atención psicosocial acordes a las necesidades de la población víctima.

Según Bueno (citado en Alvis, 2009) el aspecto psicosocial nace por situaciones "dadas por la apertura hacia el cambio social, propia de esta orientación, la cual está constantemente estudiando la forma en que los cambios sociales acelerados influyen en la interacción entre el sujeto y su medio social concreto" (p.3); así mismo, por "la demanda efectuada 
desde un enfoque psicosocial para aplicar desde su particularidad sobre las manifestaciones" (p.3).

Así mismo, Alvis (2009) plantea que la intervención con enfoque psicosocial promueve la intervención social y comunitaria, desde una perspectiva mediadora entre las víctimas y la estructura institucional desde la que se interviene, en la que participa activa y constructivamente el usuario; afirmando que esta intervención "está enmarcada en el ámbito de los derechos humanos y sociales de los ciudadanos" (p.4), buscando el de desarrollo de capacidades y potencialidades de los seres humanos.

En el caso de los niños, niñas adolescentes, a través de la estimulación de su capacidad y su habilidad, se busca trasforma o moldear de su estilo de vida, ayudando en la construcción de un nuevo proyecto de vida que les ayude a tener una perspectiva saludable de su futuro.

Por otro lado, teniendo en cuenta los planteamientos de Bello \& Chaparro (2011), el enfoque psicosocial apunta a la indagación y comprensión de la experiencia humana desde su subjetividad, pero teniendo en cuenta la interacción que tiene el sujeto con las demás personas, es decir, lo psicosocial parte de preguntar por la experiencia subjetiva de una persona inscrita en espacios más amplios de relación con otros.

Ahora bien, contextualizando este enfoque al proceso restablecimiento de las víctimas del conflicto armado, como principal aspecto para mitigar los daños psicosociales causados por esta problemática sociopolítica, es definido como un "Perspectiva que reconoce los impactos psicosociales que comprometen la violación de derechos en el contexto de la violencia y el desplazamiento en Colombia." (Ministerio de Protección Social, 2009, p.9).

Por su parte, Comité Internacional de la Cruz Roja -CICR (2011) afirma que el enfoque psicosocial incluye tanto lo psicológico como la realidad situación social de los individuos de manera que se alivie el sufrimiento y se construyan los caminos para la resiliencia.

Según la UARIV, el enfoque psicosocial debe ser visto como el conjunto de acciones emprendidas para atender el sufrimiento de las víctimas, indagando sobre los elementos de los daños psicosociales que podrían ser mitigados en a través de la rehabilitación. De la misma manera expone que, la incorporación de los criterios psicosociales en la medida de rehabilitación debe estar enfocada en el restablecimiento de las víctimas, buscando que 
las víctimas logren incorporar al devenir de la historia personal y social, la capacidad de superación y de resistencia.

Por otro lado, manifiesta que:

A través de este enfoque se busca que la reparación a las víctimas atienda las formas de interpretación que ellos y ellas y las comunidades a las que pertenecen, dan a lo que les sucedió, desde su experiencia emocional, cognitiva y relacional, y al significado que dan a la reparación y al restablecimiento de sus derechos.

\section{Programa de atención psicosocial y salud integral a víctimas - PAPSIVI}

A fin de responder a las necesidades de las víctimas en cuanto a la atención en salud y psicosocial el Estado creo el Programa PAPSIVI que se define según el (Decreto 1400 de 2011, ART 164)como:

El conjunto de actividades, procedimientos e intervenciones interdisciplinarias diseñados por el Ministerio de Salud y Protección Social para la atención integral en salud y atención psicosocial. Podrán desarrollarse a nivel individual o colectivo y en todo caso orientadas a superar las afectaciones en salud y psicosociales relacionadas con el hecho victimizante.

Este programa busca mitigar el sufrimiento emocional y aportar herramientas psicosociales que ayuden a las comunidades a la reconstrucción de su proyecto de vida, el restablecimiento de la confianza y los lazos sociales.

El programa consta de dos componentes esenciales la atención psicosocial y la atención integral en salud. Solo el ultimo incluye la atención médica especializada en salud mental ya que el primero centra su atención en el daño psicosocial, que tiene un carácter más colectivo.

De acuerdo al Ministerio de Salud y Protección Social (2009) la atención psicosocial comprende:

- Búsqueda activa de las víctimas en zonas geográficas dispersas, incluyendo población retornada o reubicada (mapeo)

- Caracterización psicosocial: daño, sufrimiento, recursos y potencialidades 
- Construcción del plan de atención psicosocial individual, familiar y comunitario con las personas afectadas.

- Atención psicosocial individual, familiar y comunitaria por equipos interdisciplinarios según caracterización del daño

- Remisión cuando sea necesario a otras instituciones del Sistema General de Seguridad Social en Salud u otros sectores.

- Registro de las actividades realizadas

- Monitoreo y seguimiento

También aclara que la atención integral en salud debe realizarse con enfoque sicosocial estableciendo que se deben realizar planes individuales de atención que recojan los impactos diferenciales y las particularidades de cada persona. Las dos estrategias deben incluir acciones, además, acciones de promoción y prevención.

\section{Metodología}

La investigación fue cualitativa, de tipo acción participativa, que según Bru \& Basagoiti este tipo de investigación es un proceso que implica "conocer-actuar-transformar", facilitando una mejor comprensión de la realidad de la población, sensibilizándoles sobre sus problemáticas y movilizando a los participantes. (Pág. 3).

El Muestreo Poblacional estuvo integrada por un total 180 niños, niñas y adolescentes víctimas del conflicto armado del municipio de Sincelejo, que acudieron al Centro de Orientación a Víctimas de la Corporación Universitaria del Caribe-CECAR-

Con el fin de recolectar la información se realizaron grupos focales que según Aigneren (2006); Beck, Bryman y Futing, 2004) citado por Escobar \& Bonilla (SF) es

Un grupo de discusión, guiado por un conjunto de preguntas diseñadas cuidadosamente con un objetivo particular", es decir, un grupo focal busca que entre sus participantes y el investigador exista una adecuada interacción encamina en 
recolectar información sobre un tema específico propuesto por

el investigador.

El objetivo general de la implantación de los grupos focales fuecrear espacios de dialogo y discusión con los niños, niñas y adolescentes, a fin de intercambiar información y experiencias relacionadas con la reparación integral descrita en la Ley de Victimas o (Ley 1448 de 2011).

\section{Panorama de la atención psicosocial en niños, niñas adolescentes victimas en el municipio de Sincelejo}

Los resultados revelan que entre la población victima menor de 18 años hay un alto desconocimiento de los derechos que les asisten, en su condición de víctimas y en los contenidos de la reparación integral.

La falta de difusión se concreta en la inexistencia programas o campañas institucionales de divulgación que informen sobre los componentes, los planes, la institucionalidad y las rutas de acceso a las medidas de reparación integral contenidas en la Ley. Lo anterior, se hace más preocupante cuando se trata de las medidas de rehabilitación, ya que por diversos medios no institucionales algunos de ellos saben que las víctimas tienen derecho a la indemnización y la ayuda humanitaria.

Se destaca que la mayoría de los niños, niñas y adolescentes desconocen su condición de víctimas directas y por tanto ignoran los derechos que les asisten por tal condición. Lo anterior se explicaría en tanto, la victimización ha sido relacionada con la comisión de hechos punibles y no mirado desde las violaciones de derechos humanos, que como se sabe, no siempre están tipificados como delitos. Un ejemplo de ello es el derecho al libre desarrollo de la personalidad que afecta el normal desarrollo del individuo en crecimiento, pero que no puede ser judicializado por no estar tipificado (Jaimes, 2014).

Al ser consultados si deseaban ser beneficiarios del programa de atención psicosocial, el setenta y ocho por ciento (78\%) manifestó interés y la necesidad de ser atendidos. Lo que permite inferir que, aunque no se reconocen como víctimas directas del conflicto armado, sienten que tienen necesidades de atención en materia de salud mental y relacionamiento con el entorno. Es importante resaltar que muchos de los NNA participantes sufrieron la violencia siendo impúberes y debieron afrontar el desarraigo y el abandono de aquello que les era importante, para enfrentar el reto de 
construir nuevas amistades y crear vínculos con un nuevo entorno en los barrios periféricos de la ciudad.

El cien por ciento (100\%) de los niños, niñas y adolescentes, expresan tener rabia o rencor por las personas que les causaron daño, produciendo estados emocionales de tristeza y dolor. Estas afectaciones merecen atención prioritaria en tanto el interés superior de la niñez y la adolescencia es un principio que debe permear las acciones del Estado. Por otro lado, al no ser atendidas adecuadamente o ser desatendidas, los sentimientos de venganza posteriores podrían llevar a estos jóvenes a enlistarse en grupos armados ilegales y continuar el círculo de la violencia armada.

Es significativo que el $42,85 \%$ no tiene un prospecto de proyecto de vida, lo que incrementa la vulnerabilidad de esta población, pues al no tener metas claras, el esfuerzo por acceder a los bienes y servicios que debe proporcionar el Estado es mucho menor, afectando gravemente el desarrollo de esta persona en todos los ámbitos de su vida.

El cincuenta y siete por ciento (57\%), aspiran llegar a ser profesionales, tal como se cita; "también, quiero ser profesional cuando sea grande". Si bien este grupo tiene claras metas, estas solo serán alcanzables si el Estado responde adecuadamente a las necesidades de superación del dolor y la rabia producto de las pérdidas económicas, familiares, sociales, culturales y afectivas.

Teniendo en cuenta los resultados del grupo focal, se realizaron entrevistas a las diferentes instituciones obligadas a prestar la atención psicosocial a las víctimas del conflicto armado a fin de contrastar la información, entre ellas, la Secretaria de Salud Municipal y la Unidad de Víctimas, esta última con el Programa de Atención Psicosocial a Víctimas (PAPSIVI). Se identificó que el municipio no cuenta con un programa de atención psicosocial que responda a las necesidades de atención diferenciada de los NNA. Los voceros de estas entidades expresan que no se delimita a la población en los programas ofrecidos; su cobertura engloba a la población en general y no existe un programa que enfatice la atención psicosocial en niños, niñas y adolescentes.

Lo anterior resulta contrario a las estipulaciones de la Corte Constitucional (2008) que hace seguimiento a la Sentencia T-025 de 2004, cuando plantea que para "un bebe, niño, niña o adolescente, la experiencia 
vital del desplazamiento forzado es intrínsecamente distinta de lo que puede ser para un hombre o una mujer adultos" (p. 13); por lo cual, establece la necesidad de adoptar un enfoque diferencial para la atención integral de los menores de edad en situación de desplazamiento; aspectos que son recogidos por la Ley de Victimas en su artículo 13, que a su vez se sustenta en El Conjunto de Principios para la protección y la promoción de los derechos humanos mediante la lucha contra la impunidad (ONU, 2005 ).

En materia de obligaciones frente a los derechos humanos y el restablecimiento de los derechos de la niñez y la adolescencia el cumplimiento es ineludible e impostergable, sin embargo, en el silencio de los que no tienen voz por su condición de vulnerabilidad el Estado sistemáticamente ha resuelto incumplir. Esto se denota en que tampoco existen programas de divulgación por medios masivos de comunicación de los contenidos del derecho a la rehabilitación psicológica de la niñez y la adolescencia víctimas del conflicto a fin de que estos sean conocidos por toda la población y en todo el territorio nacional.

Lo anterior se reafirma en que el Registro Único de Víctimas, no focaliza específicamente a los NNA, sin que los incluya dentro del núcleo familiar como un integrante más sin adentrarse en las particularidades de las afectaciones sufridas directa o indirectamente por ellos y ellas. La ausencia de un diagnostico al menos cuantitativo amplia la brecha de discriminación e incrementa la invisibilizarían de las particularidades psicológicas, afectivas, emocionales y de desarrollo de esta población.

Como bien lo plantea el Auto 251 de 2008 de seguimiento a la Sentencia T- 025 de 2014, el común denominador tanto de los problemas transversales diferenciados de la niñez y la adolescencia en situación de desplazamiento, es la conjunción de graves falencias en la respuesta estatal a esta situación, hoy 10 años después de su emisión y a pesar de los contenidos de la Ley de víctimas en sus principios rectores, la satisfacción de los derechos a la reparación integral de los NNA sigue sin respuesta estatal.

La existencia del programa PAPSIVI no ha sido la prenda de garantía para las aspiraciones de restablecimiento psicológico y psicosocial de esta población puesto que en su diseño y ejecución con contiene acciones pertinentes que atiendan específicamente a los NNA, ni de manera individual, ni colectiva. El programa atiende a las comunidades desde una mirada de 
colectividad homogénea, lo que impide que afloren las particularidades de las poblaciones con especial protección; máxime cuando en la mayoría de los casos no son convocados a los encuentros comunitarios o familiares de atención psicosocial.

Por otra parte, explica que "la respuesta de las autoridades competentes a la preocupante situación psicosocial de los niños, niñas y adolescentes en situación de desplazamiento ha sido débil y mayormente ineficaz" (p, 214), y por ello, como respuesta a la situación psicosocial, el estado prende brindar esa atención a través de existencia de unidades móviles del Instituto Colombiano de Bienestar Familiar, ICBF; sin embargo, se encontró que la población indagada no ha sido atendida por estas unidades móviles.

Por otro lado, el mismo Auto de seguimiento (Corte Constitucional Auto de seguimiento 251 de 2008, 2008) evidenció el desinterés del sistema educativo en proveer atención psicosocial a sus estudiantes en situación de desplazamiento. Deacuerdo a los resultados estas circunstanciasse mantienen no obstante los llamados de la Corte Constitucional a intervenir en favor de los NNA. Todos los participantes están escolarizados en instituciones públicasque tienenprofesionales en psicología y trabajo.

\section{Conclusiones}

Una política reparación integral que no parte de criterios claros para determinar la dimensión del daño sufrido por la víctima desconoce los principios de enfoque de derechos humanos y diferencial. No incluir de manera autónoma los derechos de los NNA en el Registro Único de víctimas niega materialmente el derecho a la reparación integral de esta población.

Establecer la calidad de la víctima es fundamental para la comprensión la condición personal de esta frente a las necesidades de rehabilitación psicológica y psicosocial, esto implica identificar la especificidad del daño a fin de satisfacer la necesidad de atención y restablecimiento que, de ser necesario, incluye el tratamiento psicológico individual y familiar del NNA.

Los obstáculos observados en cuanto al cumplimiento de la política pública de atención diferencial a la niñez y la adolescencia no son del orden local, todo lo contrario, responde a un diseño e implementación viciado desde 
el orden nacional que no responde a los estándares de integralidad, dignidad y transformadores de la reparación.

La no atención psicológica y psicosocial de los NNA víctimas, no solo violenta sus derechos fundamentales a ser protegidos por el Estado, sino que allana el camino a la repetición de los hechos violentos, pues al no ser atendidas las necesidades de canalización y manejo de las emociones y los dolores, los sentimientos reprimidos podrían dar paso a la venganza.

\section{Referencias}

Alvis. A (2009). Aproximación teórica a la intervención psicosocial. Revista Electrónica de Psicología Social «Poiésis» ISSN 1692-0945. N 17

ANSPE. (2013). Enfoque diferencial. Power point. Recuperado de: http:// www.anspe.gov.co/sites/default/files/imce/Memorias/7._presentacion_enfoque_diferencial_-_18sep.pdf

Bello. M \& Chaparro. P. (2011) El Daño desde el Enfoque Psicosocial. Recuperado de www.bivipas.info/.../L-120-Bello_Nubia_Chaparro_Ricardo-2011-458.p...

Bru \& Basagoiti. (Sf). La Investigación-Acción Participativa como metodología de mediación e integración socio-comunitaria. Recuperado de http://www.pacap.net/es/publicaciones/pdf/comunidad/6/ documentos_investigacion.pdf

Comité Internacional de la Cruz Roja (2011). Los niños afectados por los conflictos armados y otras situaciones de violencia. Recuperado de https://www.icrc.org/spa/assets/files/publications/icrc-003-4082. pdf

Consejo Económico y Social de Las Naciones Unidas (2005) Resolución 2005/102. Conjunto de principios actualizado para la protección y la promoción de los derechos humanos mediante la lucha contra la impunidad.

Corte Constitucional de Colombia (2008) Auto No 251 de 2008.Sala Segunda de Revisión

Corte IDH, Caso Alobotoe y otros, Reparaciones, Serie C, No. 15, sentencia del 10 de septiembre de 1993, párr. 43. Recuperad http://www.corteidh.or.cr/docs/casos/articulos/seriec_15_esp.pdfo de

Comite Internacional De La Cruz Roja Cicr (2011). 
Corte Constitucional Auto de seguimiento 251 de 2008, sentencia T-025 de 2004 (2008).

Corte Constitucional Sentencia T 045/10 De (2009).

Corporación AVRE. (2006). Aspectos psicosociales de la reparación integral. Recuperado de: http://www.corporacionavre.org/files/pdf/ psicosocial.pdf

Decreto 1400 De 2011 (Art 164)

Escobar \& Bonilla. (Sf). Grupos focales: una guía conceptual y metodológica. Recuperado de http://www.uelbosque.edu.co/sites/default/files/publicaciones/revistas/cuadernos_hispanoamericanos_psicologia/volumen9_numerol/articulo_5.pdf

Hewitt. N, Gantiva. C, Vera. A, Cuervo. M \& Hernández. N. (2014). Afectaciones psicológicas de niños y adolescentes expuestos al conflicto armado en una zona rural de Colombia. Acta.colomb.psicol. 17 (1): 79-89. doi: 10.14718/ACP.2014.17.1.9

Jaimes, M. (2013). El Derecho a la Reparación Individual de niñas niños y adolescentes. ¿Una utopía? Propuesta de investigación elaborada en la Corporación Universitaria del Caribe CECAR

Ley 1448 de 2011. Por la cual se dictan medidas de atención, asistencia y reparación integral a las víctimas del conflicto armado interno y se dictan otras disposiciones. Junio 10 de 2011. DO: 48096. (Colombia)

Ministerio de Protección Social. (2009). Enfoque psicosocial en la atención a la población desplazada, confinada o en riesgo. Guía práctica para Alcaldes y Alcaldesas.

Observatorio de Construcción de paz. (2012). Identidades, enfoque diferencial y construcción de paz. Recuperado de http://www. utadeo.edu.co/files/collections/documents/field_attached_file/ serie3_1.pdf

Organización Internacional para las Migraciones (OIM). (2012). Pautas para mejorar el bienestar psicosocial de las poblaciones migrantes y en situación vulnerable. Recuperado de http://www.plataformadeaprendizaje.iom.int/public/documentos/pautas_bienestar_psicosocial.pdf 
Puentes.J. (2012).La Incidencia del Programa de Atención Especializada a Niños, Niñas y Adolescentes. Facultad latinoamericana de ciencias sociales. México.

Sánchez, A (2002). Psicología social aplicada. Recuperado de http://books. google.com.co/books?id=W5327FlmSHAC\&pg=PA9\&dq=enfoque+psicosocial\&hl=es-419\&sa=X\&ei=vTwCVK71LYWMyATUiIDACw\&ved=0CB4Q6AEwAQ\#v=onepage\&q=enfoque\%20psicosocial $\& \mathrm{f}=$ false

Toro, D. (2011). Acompañamiento psicosocial a niños y niñas víctimas del conflicto armado en Colombia: un escenario para la acción sin daño. Recuperado de http://bivipas.info/bitstream/10720/570/1/ TT-134-Toro_Diana-2011-439.pdf

Unidad para la Atención y Reparación Integral a las Víctimas (S.F) Enfoque Psicosocial: Directrices del enfoque psicosocial orientadas a los planes, acciones y programas de Atención, Asistencia y Reparación a Víctimas. Recuperado de www.valledelcauca.gov.co/ salud/descargar.php?id=14061

Unicef (2013). Impacto del conflicto armado en el estado psicosocial de niños, niñas y adolescente. Recuperado de www.unicef.com. co/.../resumenejecutivo_investigacionestado_psicosocial

Villalba. C. (2004). El concepto de resiliencia. Aplicaciones en la intervención social. Recuperado de: http://www.addima.org/Documentos/Articulos/Articulo\%20Cristina\%20Villalba\%20Quesada.pdf 